\section{Prevalência de dislipidemia em adultos de meia-idade com polimorfismo do gene NOS3 e baixa aptidão cardiorrespiratória}

\author{
Prevalence of dyslipidemia in middle-aged adults with NOS3 \\ gene polymorphism and low cardiorespiratory fitness
}

Pamella A. Malagrino', Carlos H. G. Sponton', Rodrigo D. Esposti', Carla F. Franco-Penteado², Romulo A. Fernandes', Marcos André C. Bezerra², Dulcinéia M. Albuquerque², Cynara M. Rodovalho ${ }^{3}$, Maurício Bacci ${ }^{3}$, Angelina Zanesco'

\section{RESUMO}

Objetivo: Analisar a influência da associação dos polimorfismos do gene da sintase do óxido nítrico endotelial (NOS3) para as posições -786T>C, Glu298Asp e íntron 4b/a e a aptidão cardiorrespiratória sobre as concentrações de nitrito/nitrato, pressão arterial, perfil lipídico e prevalência de doenças cardiometabólicas em adultos. Sujeitos e métodos: Noventa e duas pessoas foram divididas de acordo com o genótipo: não polimórficas (NP) e polimórficas (P). Posteriormente, foram subdivididas pela aptidão cardiorrespiratória associada ao genótipo: alta (ANP e AP) ou baixa (BNP e BP). Resultados: Os indivíduos que apresentavam polimorfismo para as posições Glu298Asp+Íntron 4b/a e Glu298Asp+-786T>C e baixa aptidão cardiorrespiratória apresentaram maiores valores de colesterol total e maior prevalência de dislipidemia. Conclusão: Nossos dados demonstram que os polimorfismos do gene da NOS3 para essas duas associações influenciam os níveis de colesterol plasmático, e essa associação foi mais claramente observada quando os indivíduos apresentavam menor nível de aptidão cardiorrespiratória. Arq Bras Endocrinol Metab. 2013;57(1):33-43

\section{Descritores}

Polimorfismos; aptidão cardiorrespiratória; dislipidemia; adultos

\begin{abstract}
Objective:To evaluate the influence of the interaction between endothelial nitric oxide synthase gene (NOS3) polymorphisms at positions -786T>C, Glu298Asp and intron 4b/a, and cardiorespiratory fitness on plasma nitrite/nitrate levels, blood pressure, lipid profile, and prevalence of cardiometabolic disorders. Subjects and methods: Ninety-two volunteers were genotyped for NOS3 polymorphisms at positions (-786T>C and Glu298Asp) and (intron 4b/a) and divided according to the genotype: non-polymorphic (NP) and polymorphic (P). After that, they were subdivided according to the cardiorespiratory fitness associated with genotype: high (HNP and HP) and low (LNP and LP). Results: The subjects with polymorphism for the interactions at positions Glu298Asp + intron 4b/a, and Glu298Asp+-786T>C showed the highest values in total cholesterol, as well as dyslipidemia. Conclusion: Our findings show that NOS3 gene polymorphisms at positions -786T>C, Glu298Asp, and intron 4b/a exert negative effects on the lipid profile compared with those who do not carry polymorphisms. Arq Bras Endocrinol Metab. 2013;57(1):33-43
\end{abstract}

\section{Keywords}

Polymorphism; cardiorespiratory fitness; dyslipidemia; adults
'Laboratório de Fisiologia Cardiovascular e Atividade Física, Instituto de Biociências, Universidade Estadual Paulista (Unesp), Rio Claro, SP, Brasil ${ }^{2}$ Centro de Hematologia e Hemoterapia, Faculdade de Ciências Médicas, Universidade Estadual de Campinas (FCMUnicamp), Campinas, SP, Brasil ${ }^{3}$ Laboratório de Evolução Molecular, Instituto de Biociências, Universidade Estadual Paulista (Unesp), Rio Claro, SP, Brasil

Departamento de Educação Física, Instituto de Biociências, Unesp, Rio Claro, SP

Correspondência para: Angelina Zanesco Departamento de Educação Física Av, 24 A, 1515

13506-900 - Rio Claro, SP, Brasil azanesco@rc.unesp.br

Recebido em 2/Mar/2012 Aceito em 14/Dez/2012 


\section{INTRODUÇÃO}

$\mathrm{O}$ óxido nítrico (NO) formado a partir da conversão da L-arginina pela ação da enzima sintase do óxido nítrico endotelial (eNOS) possui papel central no controle do sistema cardiovascular. Além disso, o NO desempenha importante função na prevenção das doenças cardiometabólicas $(1,2)$. Assim, diminuição da produção do NO pelas células endoteliais e/ou sua menor biodisponibilidade para os tecidos, fenômeno caracterizado por disfunção endotelial, podem acarretar importantes alterações metabólicas bem como alterações no estado redox (aumento do estresse oxidativo), contribuindo para a gênese e o desenvolvimento de doenças como a dislipidemia e a aterosclerose (3). Os mecanismos pelos quais ocorre menor produção de $\mathrm{NO}$ e/ou sua biodisponibilidade têm sido associados a alterações de glicemia, perfil lipídico, dieta hipercalórica e sedentarismo, entre outros (4). Mais recentemente, alterações genéticas (polimorfismo) no gene da enzima eNOS (NOS3) foram associadas à disfunção endotelial, mostrando que a presença de polimorfismo no gene da eNOS poderia ser um fator desencadeador de doenças cardiometabólicas (5).

Entre os polimorfismos do gene da eNOS, os mais estudados e clinicamente relevantes são os polimorfismo caracterizados pela substituição de um único nucleotídeo (single nucleotide polymorphism - SNP), SNPs da região promotora $(-786 \mathrm{~T}>\mathrm{C})$, o polimorfismo do éxon 7 (Glu298Asp) e o VNTR localizado no íntron 4 com $27 \mathrm{pb}$ repetidos (íntron $4 \mathrm{a} / \mathrm{b}$ ) (6). De fato, estudos prévios demonstraram que os polimorfismos do gene da NOS3 para as posições $-786 \mathrm{~T}>\mathrm{C}$, caracterizado pela substituição da Timina (T) para citosina (C) na região promotora do gene; $894 \mathrm{G}>\mathrm{T}$ (Glu298Asp), caracterizado pela substituição do aminoácido glutamato (Glu) para aspartato (Asp) na enzima eNOS e íntron $4 \mathrm{~b} / \mathrm{a}$, caracterizado por quatro repetições em tandem de $27 \mathrm{pb}$, estão associados ao desenvolvimento de doenças cardiovasculares como hipertensão arterial, doença isquêmica cardíaca e acidente vascular encefálico, bem como doenças endocrinometabólicas $(7,8)$.

A prática regular de exercício físico aeróbio de moderada intensidade promove efeitos benéficos tanto na prevenção quanto no tratamento das doenças cardiometabólicas, e esses efeitos foram associados à maior produção de $\mathrm{NO}$ e/ou sua biodisponibilidade (4). Assim, sabe-se que o aumento do fluxo sanguíneo desencadeado pelo exercício físico é um poderoso estímulo para a produção de NO pelas células endoteliais e/ou aumento de sua biodisponibilidade pela maior atividade de enzimas antioxidantes $(9,10)$.

Estudos prévios avaliaram a relação entre a presença de polimorfismos do gene da NOS3 e a prática de atividade física (11), o nível de aptidão cardiorrespiratória (12), bem como o efeito do treinamento físico (13-16). No entanto, apenas um trabalho avaliou a influência do polimorfismo do gene da eNOS sobre parâmetros metabólicos em indivíduos treinados (16). Além disso, até o presente momento nenhum estudo avaliou a interação entre os polimorfismos do gene da NOS3 (-786T>C, Glu298Asp e íntron 4b/a) e o nível de aptidão cardiorrespiratória. Portanto, o objetivo do presente estudo foi verificar a influência da interação entre os polimorfismos do gene NOS3 para as posições -786T >C, Glu298Asp e íntron 4b/a sobre os parâmetros cardiovasculares e endocrinometabólicos em adultos de meia-idade. Avaliamos também a influência dos genótipos sobre a prevalência de doenças cardiometabólicas e de que forma a aptidão cardiorrespiratória poderia influenciar os diferentes parâmetros bioquímicos, como perfil lipídico, glicemia e concentração plasmática de nitrito/nitrato.

\section{SUJEITOS E MÉTODOS}

\section{Sujeitos}

O presente estudo foi aprovado pelo Comitê de Ética em pesquisa com seres humanos do Instituto de Biociências da Universidade Estadual Paulista (Unesp/Rio Claro; Protocolo 6603). Os critérios de inclusão foram: idade $\geq 40$ anos, não fumante, função renal normal (creatinina $<1,4 \mathrm{mg} / \mathrm{dL}$ ) e índice de massa corporal $<34,9 \mathrm{~kg} / \mathrm{m}^{2}$. Os critérios de exclusão foram: presença de doenças cardiovasculares (angina, doença valvular e AVE); consumo de álcool $\geq 3$ drinks por dia; problemas ortopédicos ou qualquer outra condição médica que influenciasse o desenvolvimento do estudo.

\section{Antropometria, pressão arterial, perfil lipídico e glicemia}

A massa corporal e a estatura foram medidas, e o índice de massa corporal (IMC) foi calculado pela divisão da massa corporal pela altura ao quadrado. A circunferência abdominal foi medida com uma fita metálica no ponto médio entre a crista ilíaca e a última costela. A pressão arterial sistólica (PAS) e pressão arterial dias- 
tólica (PAD) foram mensuradas com esfigmomanômetro aneroide (Tycos, USA). Após quinze minutos de repouso na posição sentada, as mensurações foram realizadas.

Após jejum de 12 horas, as amostras de sangue foram obtidas da veia antecubital $(6 \mathrm{ml})$. A lipoproteína de baixa densidade - LDL-C (Wiener, Rosário, Argentina), lipoproteína de alta densidade - HDL-C, colesterol total - CT, triglicerídeos - TG, creatinina e glicemia foram avaliados enzimaticamente por meio de kits comerciais (LaborLab, São Paulo, Brazil).

\section{Concentrações plasmáticas de nitrito/nitrato}

Amostras de plasma foram utilizadas para determinação da produção endógena de $\mathrm{NO}$ por meio da quantificação dos ânions nitrito $\left(\mathrm{NO}_{2}^{-}\right)$e nitrato $\left(\mathrm{NO}_{3}^{-}\right)$, produtos terminais da oxidação do NO. As amostras foram ultrafiltradas por meio de microfiltros (Microcon Centrifugal Filter Units, $10 \mathrm{kDa}$; Millipore, Bedford, MA, USA). Após esse procedimento, as concentrações de nitrito/nitrato (NOX) foram determinadas pelo kit comercial (Cayman Chemical, Ann Arbor, MI, USA) seguindo as instruções do fabricante. As absorbâncias foram determinadas em ELISA com comprimento de onda $(\lambda)$ de $540-550 \mathrm{~nm}$. O coeficiente de variação do método é de $3,4 \%$ entre as amostras, e entre as duplicatas é de $2,7 \%$. A sensibilidade do método é de $2,5 \mu \mathrm{M}$.

\section{Aptidão cardiorrespiratória}

$\mathrm{O}$ consumo máximo de oxigênio $\left(\mathrm{VO}_{2} \max \right)$ foi determinado por meio do teste de uma milha de Rockport (17), o qual foi utilizado para a estratificação da aptidão cardiorrespiratória da população estudada. $\mathrm{O}$ nível de aptidão cardiorrespiratória foi determinado. Os participantes foram assim divididos após o teste de uma milha: AP - Moderada/Alta aptidão cardiorrespiratória; BNP - Regular/Baixa aptidão cardiorrespiratória.

\section{Análises genotípicas}

O DNA genômico foi extraído dos leucócitos pelo método fenol-clorofórmio. A reação em cadeia da polimerase (PCR) foi realizada com 1 ciclo de $96^{\circ} \mathrm{C}$ por 2 minutos, seguido por 35 ciclos repetidos de desnaturação a $96^{\circ} \mathrm{C}$ por 30 segundos, anelamento a $60^{\circ} \mathrm{C}$ por 30 segundos e extensão a $72^{\circ} \mathrm{C}$ por $\mathrm{l}$ minuto, terminando com uma extensão a $72{ }^{\circ} \mathrm{C}$ por 5 minutos para identificação dos polimorfismos da região promotora -786T >C, do éxon 7 Glu298Asp e do íntron $4 \mathrm{~b} / \mathrm{a}$. Todas as avaliações genéticas foram realizadas no Laboratório do Professor Dr. Fernando Costa, Hemocentro da Universidade Estadual de Campinas.

\section{$-786 \mathrm{~T}>\mathrm{C}$}

A região promotora $-786 \mathrm{~T}>\mathrm{C}$ do gene da eNOS foi amplificada com a utilização de primers sense: 5'CACCCAGGCCCACCCCAACT 3'; e antisense: 5' GCCGCAGGTCGACAGAGAGACT 3'. Após a amplificação, os fragmentos foram digeridos por 5 minutos a $37^{\circ} \mathrm{C}$ utilizando $2 \mathrm{uL}$ da enzima de restrição FastDigest ${ }^{\circledR}$ MspI seguida pela eletroforese de 3 horas em gel $(2,5 \%)$ de agarose. $\mathrm{O}$ alelo $\mathrm{T}$ gera um fragmento de $394 \mathrm{pb}$ e o alelo C gera fragmentos de 352 e $42 \mathrm{pb}$.

\section{Glu298asp}

A amplificação do éxon 7 do gene da eNOS Glu298Asp foi realizada por meio da utilização de primers: sense: 5' AAGGCAGGAGACAGTGGATGGA 3'; e antisense: 5' CCCAGTCAATCCCTTTGGTGCTCA 3' (18). Após a amplificação, os fragmentos foram digeridos overnight a $37^{\circ} \mathrm{C}$ usando $2 \mathrm{uL}$ da enzima de restrição MboI seguida pela eletroforese de 3 horas em gel $(2,5 \%)$ de agarose. $\mathrm{O}$ alelo $\mathrm{G}$ gera um fragmento de $248 \mathrm{pb}$ e o alelo T gera fragmentos de 190 e 58 pb.

\section{Întron 4 b/a}

A amplificação dos fragmentos de $27 \mathrm{pb}$ em tandem do íntron $4 \mathrm{~b} / \mathrm{a}$ foi realizada por meio da utilização de primers: sense, 5' -FAM-AGGCCCTATGGTAGTGCCTTG- 3'; e antisense, 5' -TCTCTTAGTGCTGTGGTCACAG- 3' (18), modificado. A genotipagem foi realizada pelo sequenciador MegaBACE ${ }^{\mathrm{TM}}$ e os resultados foram analisados pelo software Fragment Profiler version 1.2. O alelo $4 \mathrm{a}$ gera um fragmento de $393 \mathrm{pb}$ e o alelo $4 \mathrm{~b}$, um fragmento de $420 \mathrm{pb}$. As análises genotípicas foram feitas de maneira duplo-cega. Os participantes foram então classificados como: polimórfico - presença de polimorfismos em um ou ambos os alelos para as posições -786T>C, Glu298Asp e íntron 4b/a agrupadas em pares; e não polimórfico - ausência de polimorfismos em ambos os alelos para as posições $-786 \mathrm{~T}>\mathrm{C}$, Glu298Asp e íntron 4b/a agrupadas em pares.

Após as avaliações cardiorrespiratória e genotípicas, os grupos foram subdivididos conforme o esquema a seguir: 

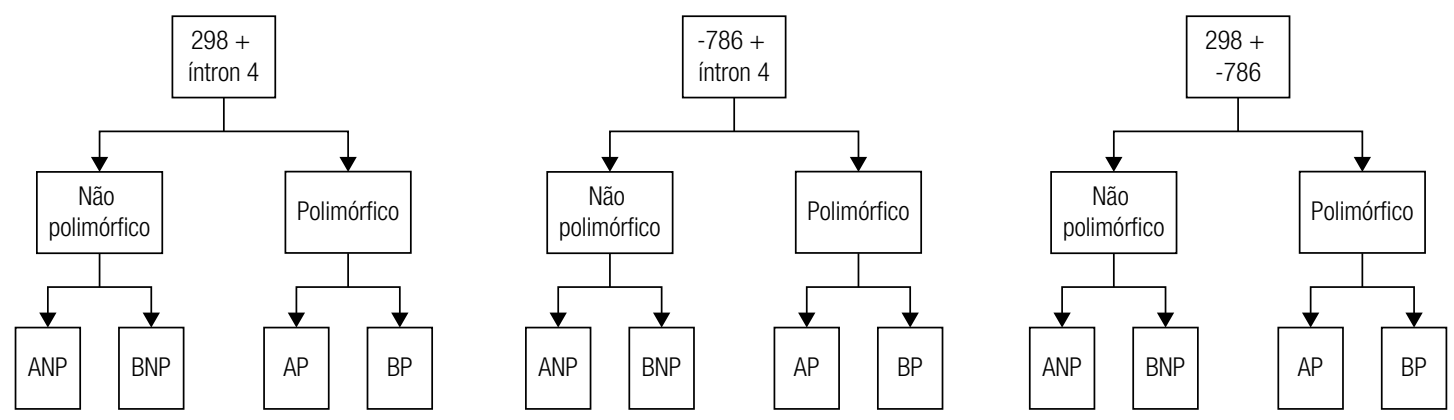

ANP: moderada/alta aptidão cardiorrespiratória e ausência de polimorfismos em ambos os alelos para as posições -786T>C, Glu298Asp e íntron 4b/a agrupadas em pares; AP: moderada/alta aptidão cardiorrespiratória e presença de polimorfismos em um ou ambos os alelos para as posições -786T>C, Glu298Asp e íntron 4b/a agrupadas em pares; BNP: regular/baixa aptidão cardiorrespiratória e ausência de polimorfismos em ambos os alelos para as posições -786T>C, Glu298Asp e íntron 4b/a agrupadas em pares; BP: regular/baixa aptidão cardiorrespiratória e presença de polimorfismos em um ou ambos os alelos para as posições -786T>C, Glu298Asp e íntron 4b/a agrupadas em pares.

\section{Análise estatística}

A análise descritiva para as variáveis contínuas foi determinada por valores de tendência central e indicadores de dispersão. Para determinação da normalidade amostral, foi utilizado o teste de Kolmogorov-Smirnov. Os valores foram expressos como média \pm erro-padrão da média (EPM), respectivamente. A distribuição dos genótipos para cada polimorfismo (equilíbrio de HardyWeinberg) e patologias como hipertensão arterial, diabetes e dislipidemia foram avaliadas utilizando o teste de Qui-quadrado $\left(\mathrm{x}^{2}\right)$. $\mathrm{O}$ teste $t$ de Student não pareado foi utilizado para comparar todas as variáveis entre os grupos não polimórficos e polimórficos. A análise de variância (ANOVA) one way seguida pelo post-hoc de Tukey foi utilizada para comparação entre os grupos ANP, AP, BNP e BP. $\mathrm{P}<0,05$ foi aceito como estatisticamente significativo.

\section{RESULTADOS}

\section{Características gerais dos participantes}

Noventa e dois voluntários (sessenta e nove mulheres e vinte e três homens) com idade média de 55,2 anos participaram do estudo. Em relação aos parâmetros antropométricos, a população estudada foi classificada com sobrepeso, apresentando valores de circunferência abdominal dentro dos limites recomendados (19). Do mesmo modo, os valores de pressão arterial, da glicemia e o perfil lipídico apresentaram valores de acordo com os limites recomendados (19) em todos os voluntários. Do total dos 92 participantes, 32 participantes apresentavam hipertensão arterial, 74 apresentavam dislipidemia e 8 sujeitos, diabetes melito. A tabela 1 sumariza esses dados.
Tabela 1. Características gerais dos voluntários do estudo

\begin{tabular}{lc}
\hline Parâmetros & (n = 92) \\
\hline Idade (anos) & $55,2 \pm 0,9$ \\
IMC (kg/m²) & $25,9 \pm 0,3$ \\
CA (cm) & $88,6 \pm 1,0$ \\
PAS (mmHg) & $115,5 \pm 1,5$ \\
PAD (mmHg) & $74,9 \pm 1,0$ \\
VO $_{2}$ max (m//kg/min) & $28,6 \pm 0,9$ \\
Glicemia (mg/dL) & $94,0 \pm 2,7$ \\
CT (mg/dL) & $200,5 \pm 4,5$ \\
LDL-C (mg/dL) & $126,1 \pm 4,4$ \\
HDL-C (mg/dL) & $49,5 \pm 1,6$ \\
TG (mg/dL) & $131,7 \pm 7,7$ \\
NOX- $(\mu \mathrm{mol} / \mathrm{L})$ & $22,4 \pm 1,5$ \\
Creatinina $(\mathrm{mg} / \mathrm{dL})$ & $0,99 \pm 0,02$ \\
\hline
\end{tabular}

IMC: índice de massa corporal; CA: circunferência abdominal; PAS: pressão arterial sistólica; PAD: pressão arterial diastólica; $\mathrm{VO}_{2}$ max: consumo máximo de oxigênio; $\mathrm{CT}$ : colesterol total; LDL-C: lipoproteína de baixa densidade; HDL-C: lipoproteína de alta densidade; TG: triglicerídeos; NOX: concentração plasmática de nitrito/nitrato. Os dados estão expressos em média \pm EPM.

\section{Avaliação genotípica do gene da eNOS (NOS3)}

A distribuição dos genótipos para os polimorfismos do gene da eNOS seguiu o equilíbrio de Hardy-Weinberg, apresentando frequência alélica de: -786 (T: 0,40 e C: 0,13), íntron 4 (b: 0,76 e a: 0,01) e 298 (G: 0,54 e T: 0,06). Não foram observadas diferenças significativas em relação à idade para as associações Glu298Asp + íntron 4b/a; -786T $>\mathrm{C}+$ íntron 4b/a; Glu298Asp + $-786 \mathrm{~T}>\mathrm{C}$ (Tabela 2$)$.

Quanto aos parâmetros antropométricos, também não foram observadas diferenças significativas entre os grupos não polimórficos e polimórficos para o IMC e circunferência abdominal para as associações Glu298Asp + íntron 4b/a, -786T>C + íntron 4b/a; 
Tabela 2. Parâmetros antropométricos, bioquímicos e prevalência de doenças cardiometabólicas para as posições -786T>C, Glu298Asp e íntron 4b/a do gene da NOS3 em adultos

\begin{tabular}{|c|c|c|c|c|c|c|}
\hline \multirow[t]{2}{*}{ Parâmetros } & \multicolumn{2}{|c|}{ Glu298Asp e intron 4b/a } & \multicolumn{2}{|c|}{$-786 \mathrm{~T}<\mathrm{C}$ e intron $4 \mathrm{~b} / \mathrm{a}$} & \multicolumn{2}{|c|}{ Glu298Asp e $-786 \mathrm{~T}<\mathrm{C}$} \\
\hline & NP $(n=35)$ & $P(n=57)$ & $N P(n=58)$ & $P(n=34)$ & $N P(n=28)$ & $P(n=64)$ \\
\hline Idade (anos) & $53,1 \pm 1,5$ & $56,5 \pm 1,1$ & $54,3 \pm 1,7$ & $55,7 \pm 1,1$ & $52,8 \pm 1,8$ & $56,3 \pm 1,1$ \\
\hline IMC $\left(\mathrm{kg} / \mathrm{m}^{2}\right)$ & $25,6 \pm 0,6$ & $26,1 \pm 0,4$ & $26,0 \pm 0,6$ & $25,9 \pm 0,4$ & $25,8 \pm 0,6$ & $26,0 \pm 0,4$ \\
\hline $\mathrm{CA}(\mathrm{cm})$ & $86,6 \pm 1,7$ & $89,9 \pm 1,2$ & $88,0 \pm 1,9$ & $89,0 \pm 1,1$ & $87,0 \pm 1,9$ & $89,3 \pm 1,1$ \\
\hline Glicemia (mg/dL) & $90,5 \pm 3,8$ & $96,2 \pm 3,6$ & $91,3 \pm 4,2$ & $95,7 \pm 3,5$ & $92,1 \pm 4,6$ & $94,9 \pm 3,3$ \\
\hline $\mathrm{TG}(\mathrm{mg} / \mathrm{dL})$ & $117,8 \pm 7,6$ & $140,3 \pm 11,5$ & $123,8 \pm 7,2$ & $136,4 \pm 11,6$ & $120,9 \pm 7,5$ & $136,5 \pm 10,6$ \\
\hline $\mathrm{HDL}-\mathrm{C}(\mathrm{mg} / \mathrm{dL})$ & $53,1 \pm 2,8$ & $47,3 \pm 1,8$ & $52,7 \pm 2,7$ & $47,6 \pm 1,9$ & $53,1 \pm 3,1$ & $47,9 \pm 1,8$ \\
\hline $\mathrm{VO}_{2} \max (\mathrm{ml} / \mathrm{kg} / \mathrm{min})$ & $31,2 \pm 1,7$ & $27,0 \pm 0,9$ & $31,2 \pm 1,7$ & $27,0 \pm 0,9$ & $31,0 \pm 1,7$ & $27,6 \pm 1,0$ \\
\hline \multicolumn{7}{|l|}{ Patologias (\%) } \\
\hline Hipertensão arterial & 22,9 & 40,4 & 32,4 & 34,5 & 32,1 & 34,4 \\
\hline Diabetes & 11,4 & 8,8 & 11,8 & 8,6 & 10,7 & 9,4 \\
\hline Dislipidemia & 68,6 & $87,7^{*}$ & 76,5 & 82,8 & 71,4 & 84,4 \\
\hline
\end{tabular}

Os dados estão expressos em média \pm EPM. Todos os dados foram analisados pelo teste $t$ de Student não pareado; *: $p<0,05$.

Glu298Asp + -786T>C. Do mesmo modo, em relação aos parâmetros bioquímicos, não foram observadas diferenças significativas para os triglicerídeos, HDL-colesterol e glicemia para as associações Glu298Asp + íntron 4b/a, -786T $>\mathrm{C}+$ íntron 4b/a e Glu298Asp $+-786 \mathrm{~T}>\mathrm{C}$. A aptidão cardiorrespiratória, valores de $\mathrm{VO}_{2} \max$, não foi diferente entre os grupos avaliados segundo o genótipo. Os dados estão sumarizados na tabela 2 .

Quanto à prevalência de doenças cardiometabólicas, não foram observadas associações significativas entre hipertensão arterial/diabetes melito tipo II e a presença de polimorfismos para as associações $-786 \mathrm{~T}>\mathrm{C}+$ íntron 4b/a e Glu298Asp + -786T>C. No entanto, uma associação significativa foi observada entre a prevalência de dislipidemia nos participantes com polimorfismo para o gene da eNOS para a associação Glu298Asp + íntron $4 \mathrm{~b} / \mathrm{a}(87,7 \%)$ em comparação àqueles sem polimorfismo para essa associação $(68,6 \%)$ (Tabela 2$)$.

Os valores de pressão arterial sistólica e diastólica também não diferiram entre os genótipos analisados para o gene da eNOS (Figura 1). Por outro lado, os valores de colesterol total $(\Delta: 12,4 \% ; \Delta: 13,5 \%)$ e LDLcolesterol $(\Delta: 17,2 \% ; \Delta: 17,9 \%)$ foram significativamente maiores nos participantes com polimorfismo para o gene da eNOS para as associações Glu298Asp + íntron 4b/a e Glu298Asp + -786T >C. Nenhuma diferença foi encontrada na associação $-786 \mathrm{~T}>\mathrm{C}+$ íntron $4 \mathrm{~b} / \mathrm{a}$ para esses parâmetros bioquímicos (Figura 2).

Em relação às concentrações plasmáticas de nitrito/ nitrato $\left(\mathrm{NOX}^{-}\right)$, não foram observadas diferenças signi- ficativas entre os grupos não polimórficos e polimórficos independentemente das associações analisadas (Figura 2).
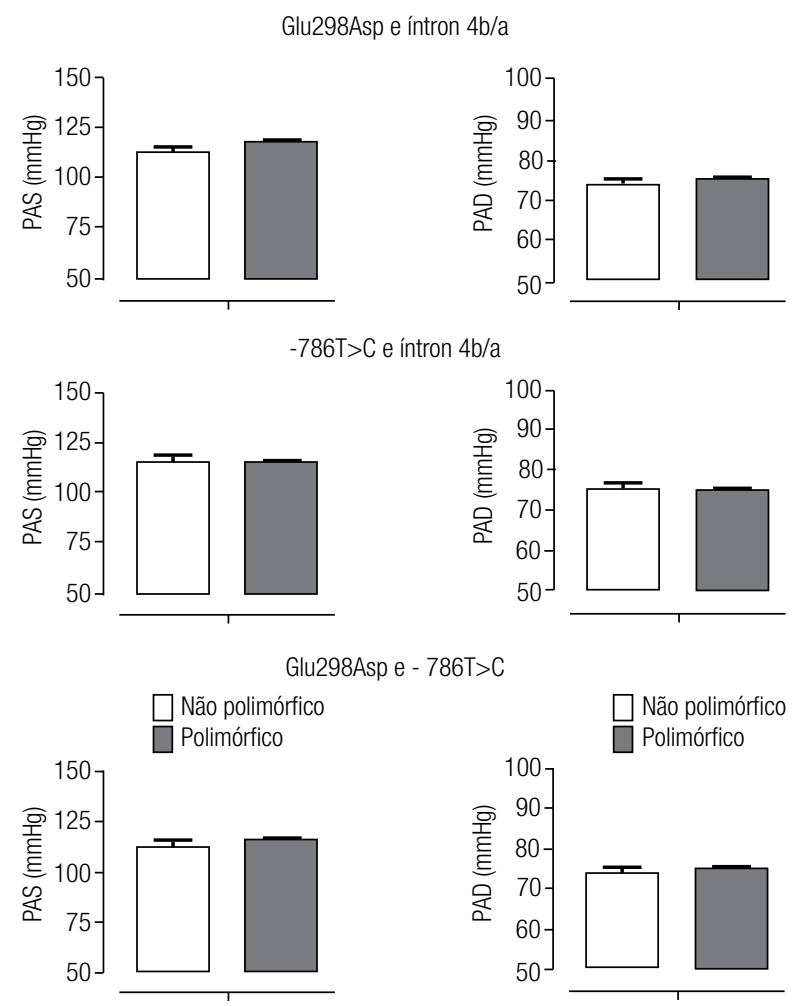

Figura 1. Valores de pressão arterial sistólica e diastólica de voluntários adultos de meia-idade subdivididos pelos polimorfismos do gene NOS3 para as posições -786T>C, Glu298Asp e íntron 4b/a. Os dados estão expressos em média \pm EPM. 
Glu298Asp e íntron 4b/a
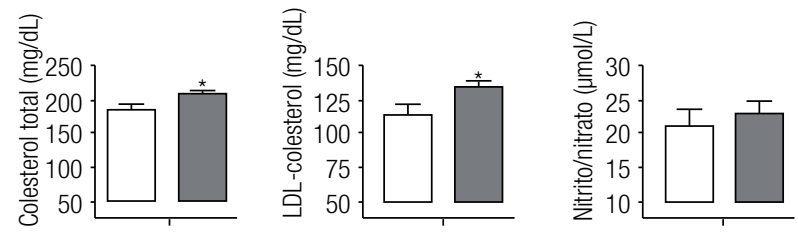

-786T >C e íntron 4b/a
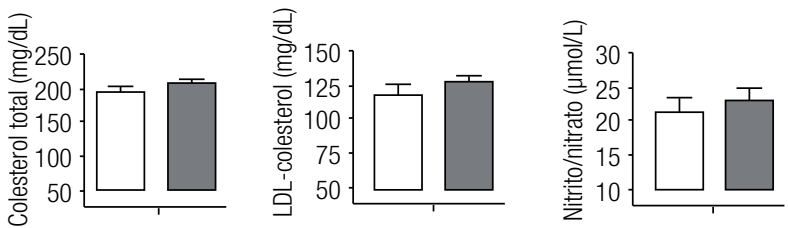

Glu298Asp e - 786T>C
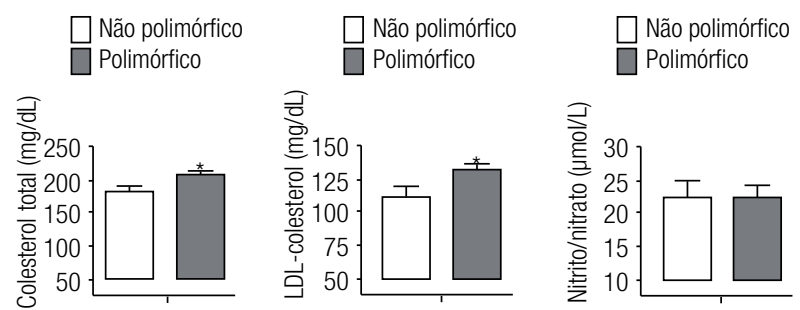

Figura 2. Níveis de colesterol total, LDL-colesterol e de nitrito/nitrato em voluntários adultos de meia-idade subdivididos pelos polimorfismos do gene NOS3 para as posições -786T>C, Glu298Asp e íntron 4b/a. Os dados estão expressos em média \pm EPM; * $p<0,05$.

\section{Associação entre genótipo Glu298Asp + íntron 4b/a e aptidão cardiorrespiratória}

A tabela 3 mostra a associação entre o genótipo Glu298Asp + íntron 4b/a e a aptidão cardiorrespiratória sobre os parâmetros antropométricos (IMC e CA), bio- químicos (glicemia, triglicerídeos e HDL-colesterol) e prevalência de doenças cardiometabólicas. Foram observadas diferenças significativas em relação à idade entre os grupos com moderada/alta (ANP) e baixa/regular (BP) aptidão cardiorrespiratória para o grupo polimórfico da associação Glu298Asp + íntron 4b/a. Em relação aos parâmetros antropométricos, os participantes com baixa aptidão cardiorrespiratória apresentaram maiores valores de IMC e CA quando comparados àqueles com maior aptidão cardiorrespiratória independente da presença de polimorfismo para o gene da eNOS para as associações Glu298Asp + íntron 4b/a (Tabela 3). Os valores de glicemia, triglicerídeos e HDL-colesterol não apresentaram qualquer diferença entre os grupos (Tabela 3).

Quanto à prevalência de patologias, observaram-se valores de associação significativa apenas para os participantes com baixa aptidão cardiorrespiratória e com polimorfismo para as posições Glu298Asp + íntron 4 b/a (grupo BP) em relação à hipertensão arterial $(43,8 \%)$ e dislipidemia $(93,8 \%)$ quando comparados aos demais grupos. Os dados estão sumarizados na tabela 3 .

Com relação à terapia farmacológica, não observamos diferença entre o uso de anti-hipertensivos entre os grupos para a associação Glu298Asp + íntron 4 b/a (ANP: 22,5; AP: 32; BNP: 36,4; BP: 31,3\%). Todavia, os participantes com baixa aptidão cardiorrespiratória $\mathrm{e}$ presença de polimorfismo para o gene da eNOS para a associação Glu298Asp + íntron 4 b/a (grupo BP) apresentaram maior porcentagem de uso de medicamentos hipolipemiantes $(21,9 \%)$ quando comparados aos demais grupos (ANP: 4,2; AP: 8; BNP: 0\%).

Tabela 3. Associação entre aptidão cardiorrespiratória e a interação das posições Glu298Asp e íntron 4b/a do gene da NOS3 sobre os parâmetros antropométricos, bioquímicos e prevalência de doenças cardiometabólicas em adultos

\begin{tabular}{|c|c|c|c|c|}
\hline & \multicolumn{4}{|c|}{ Glu298Asp e íntron 4b/a } \\
\hline & ANP $(n=24)$ & AP $(n=25)$ & BNP $(n=11)$ & $\mathrm{BP}(n=32)$ \\
\hline Idade (anos) & $51,1 \pm 1,6$ & $55,0 \pm 1,3$ & $57,3 \pm 2,9$ & $57,7 \pm 1,7^{a}$ \\
\hline IMC $\left(\mathrm{kg} / \mathrm{m}^{2}\right)$ & $23,9 \pm 0,5$ & $25,2 \pm 0,7$ & $29,3 \pm 0,6^{a b}$ & $26,8 \pm 0,5^{\mathrm{a}}$ \\
\hline $\mathrm{CA}(\mathrm{cm})$ & $83,5 \pm 1,9$ & $88,3 \pm 1,9$ & $93,9 \pm 2,0^{\mathrm{a}}$ & $91,0 \pm 1,4^{\mathrm{a}}$ \\
\hline Glicemia (mg/dL) & $90,8 \pm 4,3$ & $98,2 \pm 5,8$ & $90,0 \pm 8,3$ & $94,7 \pm 4,7$ \\
\hline $\mathrm{TG}(\mathrm{mg} / \mathrm{dL})$ & $114,0 \pm 9,5$ & $129,7 \pm 16,5$ & $126,2 \pm 12,8$ & $148,6 \pm 16,0$ \\
\hline $\mathrm{HDL}-\mathrm{C}(\mathrm{mg} / \mathrm{dL})$ & $53,5 \pm 3,3$ & $47,1 \pm 2,8$ & $52,1 \pm 5,3$ & $47,4 \pm 2,5$ \\
\hline \multicolumn{5}{|l|}{ Patologias (\%) } \\
\hline Hipertensão arterial & 16,7 & 36 & 36,4 & $43,8^{*}$ \\
\hline Diabetes & 8,3 & 8,0 & 18,2 & 9,4 \\
\hline Dislipidemia & 70,8 & 80 & 63,6 & $93,8^{*}$ \\
\hline
\end{tabular}

ANP: moderada/alta aptidão cardiorrespiratória e ausência de polimorfismos; AP: moderada/alta aptidão cardiorrespiratória e presença de polimorfismo; BNP: regular/baixa aptidão cardiorrespiratória e ausência de polimorfismos; BP: regular/baixa aptidão cardiorrespiratória e presença de polimorfismos para as posições do gene da eNOS analisadas. Os dados estão expressos em média \pm EPM; ANOVA one way seguida pelo post-hoc de Tukey; 0 teste de $x^{2}$ foi utilizado para a associação entre as patologias e os medicamentos e os grupos ANP, AP, BNP e BP; *: $p<0,05 ;$ a: $p<0,05$ em relação ao grupo ANP; $b=p<0,05$ em relação ao grupo AP. 


\section{Associação entre genótipo -786T>C + íntron 4b/a e aptidão cardiorrespiratória}

A tabela 4 mostra a associação entre a presença ou não de polimorfismo para o gene da eNOS nas posições $-786 \mathrm{~T}>\mathrm{C}+$ íntron $4 \mathrm{~b} / \mathrm{a}$ e a aptidão cardiorrespiratória sobres os parâmetros antropométricos, bioquímicos e prevalência de doenças cardiometabólicas.

Podemos observar que os participantes com baixa aptidão cardiorrespiratória (grupo BNP e BP) apresentam maiores valores de IMC e CA quando comparados aos grupos com melhor aptidão cardiorrespiratória (grupos ANP e AP), independente da presença de polimorfismo para as posições $-786 \mathrm{~T}>\mathrm{C}+$ íntron $4 \mathrm{~b} / \mathrm{a}$ do gene da eNOS (Tabela 4).

Quando avaliada a prevalência de patologias cardiometabólicas, observamos que, para as posições $-786 \mathrm{~T}>\mathrm{C}$ + íntron $4 \mathrm{~b} / \mathrm{a}$ do gene da NOS3, a prevalência de hipertensão arterial, diabetes melito tipo II e dislipidemia não difere entre os grupos analisados (Tabela 4). No entanto, os participantes com baixa aptidão cardiorrespiratória e presença de polimorfismo (BP) apresentam maior porcentagem de uso de medicamentos hipolipemiantes $(25 \%, \mathrm{P}<0,05)$ em relação aos demais grupos (ANP: 5,3; AP: 6,7; BNP: 0\%). Não houve diferença entre os grupos para os valores de glicemia, triglicerídeos, HDLcolesterol e uso de medicação anti-hipertensiva.

\section{Associação entre genótipo Glu298Asp +-786T>C e aptidão cardiorrespiratória}

A tabela 5 mostra a associação entre a presença ou não de polimorfismo para o gene da NOS3 nas posições Glu298Asp + -786T >C e a aptidão cardiorrespiratória sobre os parâmetros antropométricos, bioquímicos e prevalência de doenças cardiometabólicas. Podemos observar que, de modo similar às avaliações feitas anteriormente, os participantes com baixa aptidão cardiorrespiratória (grupos BNP e BP) apresentam valores de IMC e circunferência abdominal (CA) maiores quando comparados àqueles com moderada/alta aptidão cardiorrespiratória (grupos ANP e AP), independente da presença de polimorfismo ou não. Nesta associação em particular, nenhuma diferença foi encontrada para os valores de glicemia, triglicerídeos, HDL-colesterol e prevalência de doenças cardiometabólicas entre os grupos estudados (Tabela 5). O uso de medicamentos anti-hipertensivos (ANP: 22,5; AP: 22,6; BNP: 40; BP: 30,3\%) e hipolipemiantes (ANP: 5,6; AP: 6,5; BNP: 10; BP: 18\%) também não foi diferente entre os grupos estudados.

A figura 3 ilustra a influência do polimorfismo do gene NOS3 associado à aptidão cardiorrespiratória sobre os valores de pressão arterial sistólica e diastólica nas três associações analisadas. Podemos observar que os participantes que possuíam baixa aptidão cardiorrespiratória apresentavam maiores valores de pressão arterial sistólica e diastólica independente do genótipo. Por outro lado, as concentrações plasmáticas de colesterol total foram maiores nos participantes que possuíam polimorfismo para o gene da NOS3 nas associações Glu298Asp + íntron4b/a e Glu298Asp + $-786 \mathrm{~T}>\mathrm{C}$ e baixa aptidão cardiorrespiratória $(\mathrm{BP}, \Delta \%=$ 15,1 e 17,3 , respectivamente) quando comparados aos outros grupos. Os níveis de LDL-colesterol e nitrito/ nitrato não foram diferentes entre os grupos analisados (Figura 4).

Tabela 4. Associação entre a aptidão cardiorrespiratória e a interação das posições -786T>C e íntron 4b/a do gene da NOS3 sobre os parâmetros antropométricos, bioquímicos e prevalência de doenças cardiometabólicas em adultos

\begin{tabular}{|c|c|c|c|c|}
\hline & \multicolumn{4}{|c|}{$-786 \mathrm{~T}>\mathrm{C}$ e íntron $4 \mathrm{~b} / \mathrm{a}$} \\
\hline & ANP $(n=19)$ & $\operatorname{AP}(n=30)$ & BNP $(n=15)$ & $\mathrm{BP}(n=28)$ \\
\hline Idade (anos) & $52,2 \pm 2,1$ & $53,6 \pm 1,1$ & $56,9 \pm 2,7$ & $57,9 \pm 1,8$ \\
\hline IMC (kg/m²) & $23,8 \pm 0,6$ & $25,0 \pm 0,6$ & $28,7 \pm 0,5^{\mathrm{ab}}$ & $26,8 \pm 0,6^{a}$ \\
\hline $\mathrm{CA}(\mathrm{cm})$ & $83,2 \pm 2,3$ & $87,7 \pm 1,6$ & $94,4 \pm 2,0^{a}$ & $90,3 \pm 1,4^{\mathrm{a}}$ \\
\hline Glicemia (mg/dL) & $90,8 \pm 5,0$ & $96,9 \pm 5,0$ & $91,8 \pm 7,2$ & $94,4 \pm 4,9$ \\
\hline $\mathrm{TG}(\mathrm{mg} / \mathrm{dL})$ & $118,9 \pm 10,6$ & $123,9 \pm 14,3$ & $130,0 \pm 9,3$ & $149,7 \pm 18,3$ \\
\hline $\mathrm{HDL}-\mathrm{C}(\mathrm{mg} / \mathrm{dL})$ & $54,2 \pm 3,7$ & $47,7 \pm 2,7$ & $50,8 \pm 3,9$ & $47,4 \pm 2,8$ \\
\hline \multicolumn{5}{|l|}{ Patologias (\%) } \\
\hline Hipertensão arterial & 21,1 & 30 & 46,7 & 39,3 \\
\hline Diabetes & 5,3 & 10 & 20 & 7,1 \\
\hline Dislipidemia & 73,7 & 76,7 & 80 & 89,3 \\
\hline
\end{tabular}

ANP: moderada/alta aptidão cardiorrespiratória e ausência de polimorfismos para as posiç̃oes do gene da eNOS analisadas; AP: moderada/alta aptidão cardiorrespiratória e presença de polimorfismos para as posições do gene da eNOS analisadas; BNP: regular/baixa aptidão cardiorrespiratória e ausência de polimorfismos para as posições do gene da NOS3 analisadas; BP: regular/baixa aptidão cardiorrespiratória e presença de polimorfismos para as posições do gene da eNOS analisadas. Os dados estão expressos em média \pm EPM; ANOVA one way seguida pelo post hoc de Tukey; 0 teste de $x^{2}$ foi utilizado para a associação entre as patologias e medicamentos e os grupos ANP, AP, BNP e BP; *: $p<0,05 ;$ a: $p<0,05$ em relação ao grupo ANP; b: $p<0,05$ em relação ao grupo AP. 
Tabela 5. Associação entre a aptidão cardiorrespiratória e a interação das posições - Glu298Asp +-786T>C do gene da NOS3 sobre os parâmetros antropométricos, bioquímicos e prevalência de doenças cardiometabólicas em adultos

\begin{tabular}{|c|c|c|c|c|}
\hline & \multicolumn{4}{|c|}{ Glu298Asp e $-786 \mathrm{~T}>\mathrm{C}$} \\
\hline & $\operatorname{ANP}(n=31)$ & AP $(n=18)$ & $\operatorname{BNP}(n=10)$ & $\mathrm{BP}(n=33)$ \\
\hline Idade (anos) & $52,6 \pm 1,4$ & $53,9 \pm 1,5$ & $56,3 \pm 3,1$ & $57,9 \pm 1,7$ \\
\hline IMC $\left(\mathrm{kg} / \mathrm{m}^{2}\right)$ & $24,1 \pm 0,6$ & $24,8 \pm 0,6$ & $28,8 \pm 0,5^{\mathrm{ab}}$ & $27,0 \pm 0,5^{a b}$ \\
\hline $\mathrm{CA}(\mathrm{cm})$ & $83,3 \pm 2,3$ & $87,5 \pm 1,7$ & $94,2 \pm 2,1^{a}$ & $91,0 \pm 1,4^{a}$ \\
\hline Glicemia (mg/dL) & $92,5 \pm 5,3$ & $95,8 \pm 4,9$ & $91,4 \pm 9,0$ & $94,1 \pm 4,6$ \\
\hline $\mathrm{TG}(\mathrm{mg} / \mathrm{dL})$ & $117,2 \pm 10,2$ & $124,7 \pm 14,1$ & $127,4 \pm 11,0$ & $147,6 \pm 15,7$ \\
\hline HDL-C (mg/dL) & $54,9 \pm 3,8$ & $47,6 \pm 2,6$ & $50,0 \pm 5,7$ & $48,2 \pm 2,5$ \\
\hline \multicolumn{5}{|l|}{ Patologias (\%) } \\
\hline Hipertensão arterial & 27,8 & 25,8 & 40 & 43,8 \\
\hline Diabetes & 5,6 & 9,7 & 20 & 9,1 \\
\hline Dislipidemia & 72,2 & 77,4 & 70 & 90,9 \\
\hline
\end{tabular}

ANP: moderada/alta aptidão cardiorrespiratória e ausência de polimorfismos para as posições do gene da eNOS analisadas; AP: moderada/alta aptidão cardiorrespiratória e presença de polimorfismos para as posições do gene da eNOS analisadas; BNP: regular/baixa aptidão cardiorrespiratória e ausência de polimorfismos para as posições do gene da eNOS analisadas; BP: regular/ baixa aptidão cardiorrespiratória e presença de polimorfismos para as posições do gene da eNOS analisadas. Os dados estão expressos em média \pm EPM; a análise de variância ANOVA one way seguida pelo post hoc de Tukey; 0 teste de $x^{2}$ foi utilizado para a associação entre as patologias e medicamentos e os grupos ANP, AP, BNP e BP; a: $p<0,05$ em relação ao grupo ANP; b: $p<0,05$ em relação ao grupo AP.

Glu298Asp e íntron 4b/a
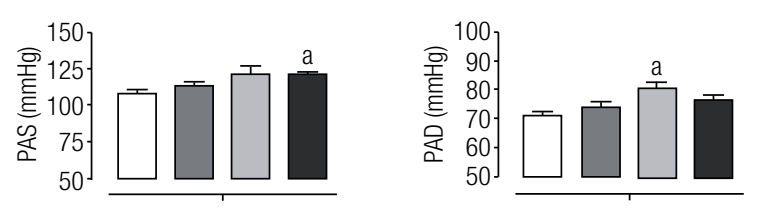

$-786 \mathrm{~T}>\mathrm{C}$ e íntron 4b/a
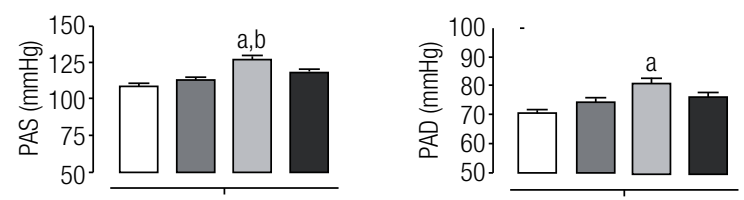

Glu298Asp e - 786T>C
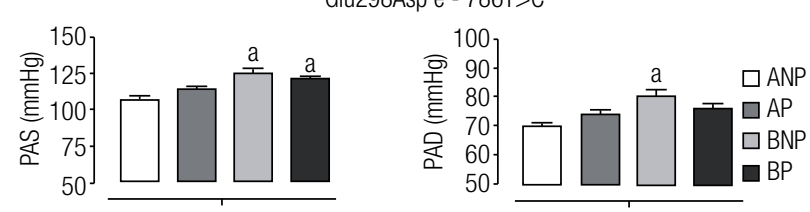

Figura 3. Valores de pressão arterial sistólica e diastólica de voluntários adultos de meia-idade divididos pelos polimorfismos do gene da eNOS para as posições -786T>C, Glu298Asp e íntron 4b/a, e subdivididos conforme avaliação da aptidão cardiorrespiratória (ANP: moderada/alta aptidão + ausência de polimorfismos para as posições -786T $>C$, Glu298Asp e íntron 4b/a; AP: moderada/alta aptidão + presença de polimorfismos para as posições -786T>C, Glu298Asp e íntron 4b/a; BNP: regular/baixa aptidão + ausência de polimorfismos para as posições -786T>C, Glu298Asp e íntron 4b/a; BP: regular/baixa aptidão + presença de polimorfismos para as posições -786T>C, Glu298Asp e íntron 4b/a). Os dados estão expressos em média \pm EPM; a: $p<0,05$ em relação ao grupo ANP; $b$ : $p<0,05$ em relação ao grupo AP.
Glu298Asp e íntron 4b/a
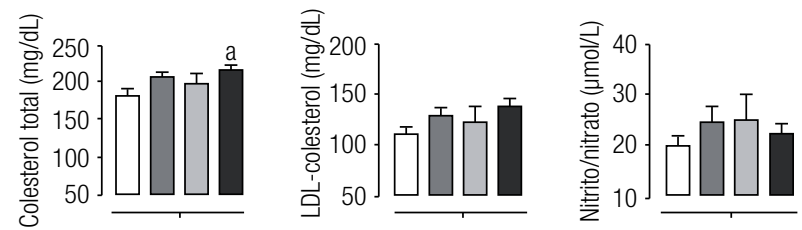

-786T>C e íntron 4b/a
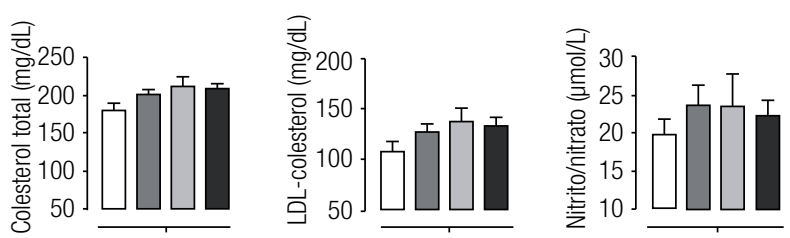

Glu298Asp e - 786T>C
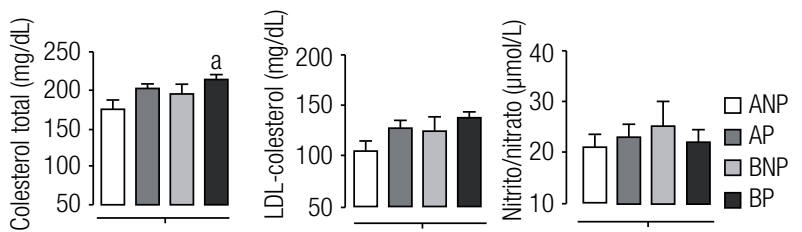

Figura 4. Níveis de colesterol total, LDL-colesterol e de nitrito/nitrato em voluntários adultos de meia-idade divididos pelos polimorfismos do gene NOS3 para as posições -786T>C, Glu298Asp e íntron 4b/a, e subdivididos conforme avaliação da aptidão cardiorrespiratória (ANP: moderada/alta aptidão + ausência de polimorfismos para as posições -786T>C, Glu298Asp e íntron 4b/a; AP: moderada/alta aptidão + presença de polimorfismos para as posições -786T>C, Glu298Asp e íntron 4b/a; BNP: regular/baixa aptidão + ausência de polimorfismos para as posições -786T>C, Glu298Asp e íntron 4b/a; BP: regular/baixa aptidão + presença de polimorfismos para as posições -786T>C, Glu298Asp e íntron 4b/a). Os dados estão expressos em média \pm EPM; a: $p<0,05$ em relação ao grupo ANP. 


\section{DISCUSSÃO}

Entre as isoformas constitutivas da enzima NO sintase (nNOS e eNOS), a eNOS (NOS3) é considerada a mais importante devido ao seu papel no controle da pressão arterial e seus efeitos antitrombóticos, por meio da sua ação enzimática sobre a L-arginina levando à formação do NO. Assim, a redução em sua expressão e/ou em sua atividade pode resultar em disfunção endotelial. Diversos fatores podem afetar a expressão e/ou a atividade de NOS3, entre eles, deficiência de cofatores para sua atividade enzimática, bem como a presença de polimorfismos no gene NOS3 (5). De fato, trabalhos prévios demonstraram que a presença de polimorfismo nas posições -786T>C, Glu298Asp e íntron 4 b/a está associada à redução na expressão e atividade da enzima $\operatorname{NOS3}(20,21)$, menor produção de nitrito/nitrato (que reflete indiretamente a produção de $\mathrm{NO}$ ), bem como menor resposta relaxante dependente do endotélio (22). No entanto, a maioria desses trabalhos avaliou essas três posições, que são consideradas clinicamente relevantes, de maneira isolada, ou seja, a presença ou não do polimorfismo do gene da NOS 3 em cada posição e sua influência nos diferentes parâmetros cardiovasculares e endocrinometabólicos. Assim, o presente trabalho buscou avaliar a interação entre a presença do polimorfismo do gene da NOS3 com parâmetros antropométricos, cardiovasculares e bioquímicos. Nossos dados demonstram que a presença dos polimorfismos do gene da NOS3 analisados para as posições $-786 \mathrm{~T}>\mathrm{C}$, Glu298Asp e íntron b/a não influencia os parâmetros antropométricos. Como esperado, os menores valores de IMC e circunferência abdominal foram encontrados nos participantes com maior aptidão cardiorrespiratória, independentemente da presença ou ausência desses polimorfismos.

Diferentemente de trabalhos prévios que observaram menores concentrações plasmáticas de nitrito/ nitrato em pacientes com polimorfismo para o gene da eNOS $(23,24)$, no presente estudo não foram observadas diferenças em relação às concentrações de nitrito/nitrato independente das associações entre os polimorfismos ou nível de aptidão cardiorrespiratória. De fato, estudo anterior realizado em nosso laboratório demonstrou que as concentrações basais de nitrito/ nitrato não diferem entre indivíduos com polimorfismo e sem polimorfismo para a posição $-786 \mathrm{~T}>\mathrm{C}$ do gene da eNOS em mulheres na pós-menopausa (15). Entretanto, quando essas mulheres foram submetidas a 24 semanas de treinamento físico aeróbio, observamos maiores incrementos nas concentrações de nitrito/nitrato e maior redução de pressão arterial para o grupo não polimórfico em comparação aos indivíduos com polimorfismo (15). Assim, confirmando resultados prévios de nosso laboratório, no qual se analisou apenas uma posição para o polimorfismo do gene da NOS3, o presente trabalho mostra que, em condições basais, a presença de polimorfismo para o gene da NOS3, combinado dois a dois, não interfere com as concentrações plasmáticas basais de nitrito/nitrato, que indiretamente refletem a produção de $\mathrm{NO}$, em adultos de meia-idade. De maneira similar, observamos que os valores de pressão arterial não foram associados à presença de polimorfismo para as posições estudadas e suas interações. No entanto, participantes com baixa aptidão cardiorrespiratória apresentaram maiores valores de pressão arterial quando comparados àqueles com maior aptidão cardiorrespiratória, independente do genótipo, reforçando a importância da prática de atividade física para a manutenção da saúde cardiovascular. De fato, estudos prévios demonstraram, de maneira sistemática, que a aptidão cardiorrespiratória é inversamente relacionada com a pressão arterial e perfil lipídico (25), sendo esse um importante e independente preditor de risco cardiovascular (26). Além disso, um estudo mostrou que um decréscimo de $5 \%$ no $\mathrm{VO}_{2}$ max resulta em $56 \%$ de incremento no risco cardiovascular (27). Por outro lado, cada $1 \mathrm{ml} / \mathrm{kg} / \mathrm{min}$ de incremento no $\mathrm{VO}_{2} \max$ resulta em uma diminuição de aproximadamente $15 \%$ no risco de morte por todas as causas (28). Portanto, nossos dados reforçam os efeitos benéficos da prática de atividade física sobre o sistema cardiovascular em indivíduos adultos de meia-idade.

Com relação ao perfil lipídico e glicemia, nosso estudo mostrou que os níveis de colesterol total e LDLcolesterol foram significativamente superiores para os grupos polimórficos em comparação àqueles que não apresentavam polimorfismo para as associações Glu298Asp + íntron 4b/a e Glu298Asp + 786T>C (maiores detalhes, veja Figura 2). Quando associamos os genótipos e o nível de aptidão cardiorrespiratória, nossos dados mostram mais claramente que indivíduos que apresentavam polimorfismo para o gene da NOS3 para as posições Glu298Asp + íntron 4b/a e Glu298Asp + 786T>C e tinham baixos níveis de aptidão cardiorrespiratória apresentam maiores valores de colesterol total, bem como maior prevalência de dislipidemias, cerca de 93,8\% (veja maiores detalhes na Figura 4 e Tabela 3). De fato, trabalhos prévios mostraram 
associação entre os polimorfismos do gene da eNOS para as posições -786T >C, Glu298Asp e íntron 4b/a e a prevalência de dislipidemia $(29,30)$. Além disso, um estudo mostrou aumento nas concentrações plasmáticas de LDL-colesterol, LDL-colesterol modificado por malondialdeído (MDA-LDL-C) e níveis de insulina em jejum em indivíduos polimórficos para a posição Glu298Asp do gene da NOS3. Esses autores verificaram também redução das concentrações de HDL-C, apoliproteína I, e consequente redução na vasodilatação dependente de endotélio (31). Assim, parece-nos que o elo comum entre dislipidemia e polimorfismo no gene da NOS3 esteja na posição Glu298Asp, onde o glutamato é substituído pelo aspartato na enzima eNOS, modificando sua atividade enzimática e/ou menor ativação por estímulos físicos, como o shear stress. De fato, um estudo observou que a presença do polimorfismo localizado no éxon 7 (Glu298Asp) resultava em alterada localização da enzima eNOS no espaço caveolar, acarretando menor resposta ao shear stress (32). Além disso, esses autores observaram que essa variação polimórfica está relacionada a uma reduzida dissociação da enzima eNOS (35\%) do complexo eNOS/ Caveolina-1, levando essa condição a uma redução da atividade enzimática e formação de nitro/nitrato. Estudo recente realizado no nosso laboratório demonstrou menor magnitude de redução do colesterol total e LDL-colesterol em resposta a oito semanas de treinamento físico aeróbio em mulheres com polimorfismo para as posições -786T >C e Glu298Asp do gene da eNOS em comparação àquelas que não apresentavam polimorfismo para essas posições, que foram estudadas de maneira isolada (16). Os possíveis mecanismos pelos quais a presença de polimorfismo para o gene da NOS3 estaria relacionada à maior prevalência de dislipidemia poderiam ser devido ao papel do NO sobre o metabolismo do colesterol. Evidências mostram que o NO possui efeitos hipocolesterolêmicos em modelos animais, nos quais se observou que a administração de doadores de NO promove significativa redução dos níveis plasmáticos de LDL-colesterol em coelhos (2). No entanto, em nosso estudo os níveis plasmáticos de nitrito/nitrato não apresentaram diferença estatística entre os grupos estudados. Diferentemente, estudos em nosso laboratório mostram que os níveis teciduais de nitrito/nitrato estão aumentados em músculo esquelético de ratos treinados, sem qualquer alteração desses metabólitos no plasma (33). Além disso, sabe-se que o músculo esquelético é um dos tecidos que mais utili- za a gordura como substrato energético, por meio da oxidação de ácidos graxos (34). Assim, nossos dados mostram claramente que a presença de polimorfismo para o gene da NOS3 (interação Glu298Asp + íntron 4b/a e Glu298Asp + 786T >C) associado à menor aptidão cardiorrespiratória determina maior prevalência de dislipidemia em adulto de meia-idade.

Em conclusão, nossos dados demonstram que os polimorfismos do gene da NOS3 para as posições -786T >C, Glu298Asp e íntron 4b/a influenciam os níveis de colesterol plasmático quando comparados aos seus congêneres não polimórficos, e essa associação foi mais claramente observada quando os indivíduos apresentavam menor nível de aptidão cardiorrespiratória. Considerando a importância das doenças cardiometabólicas (dislipidemia/aterosclerose) para o sistema de saúde, nossos resultados poderiam ser utilizados para criar estratégias para a prevenção dessas patologias, bem como para evitar as complicações advindas destas, como a doença arterial coronariana e acidente vascular encefálico, entre outras.

A principal limitação do presente estudo foi o reduzido número de voluntários constituindo cada grupo analisado. Por outro lado, seria interessante que os diferentes grupos de pesquisa existentes no país buscassem estratégias para formar bancos de dados com o objetivo de estudar grandes grupos populacionais e assim obter resultados com maior poder estatístico das análises genéticas da população brasileira, que possui características singulares no que se refere à etnia.

Agradecimentos: os autores são gratos à Fundação de Amparo à Pesquisa do Estado de São Paulo (Fapesp) pelo apoio financeiro.

Declaração: os autores declaram não haver conflitos de interesse científico neste estudo.

\section{REFERÊNCIAS}

1. Moncada S, Higgs EA. Nitric oxide and the vascular endothelium. Handb Exp Pharmacol. 2006;176:213-54.

2. Jobgen WS, Fried SK, Fu WJ, Meininger CJ, Wu G. Regulatory role for the arginine-nitric oxide pathway in metabolism of energy substrates. J Nutr Biochem. 2006;17(9):571-88.

3. Balligand $\mathrm{JL}$, Feron O, Dessy C. eNOS activation by physical forces: from short-term regulation of contraction to chronic remodeling of cardiovascular tissues. Physiol Rev. 2009;89(2):481-534.

4. Zanesco A, Antunes E. Effects of exercise training on the cardiovascular system: pharmacological approaches. Pharmacol Ther. 2007;114(3):307-17.

5. Förstermann U, Sessa WC. Nitric oxide synthases: regulation and function. Eur Heart J. 2012;33(7):829-37, 837a-837d. 
6. Casas JP, Cavalleri GL, Bautista LE, Smeeth L, Humphries SE, Hingorani AD. Endothelial nitric oxide synthase gene polymorphisms and cardiovascular disease: a HuGE review. Am J Epidemiol. 2006;164(10):921-35.

7. Sandrim VC, Yugar-Toledo JC, Desta Z, Flockhart DA, Moreno H $\mathrm{Jr}$, Tanus-Santos JE. Endothelial nitric oxide synthase haplotypes are related to blood pressure elevation, but not to resistance to antihypertensive drug therapy. J Hypertens. 2006;24(12):2393-7.

8. Sandrim VC, de Syllos RW, Lisboa HR, Tres GS, Tanus-Santos JE. Influence of eNOS haplotypes on the plasma nitric oxide products concentrations in hypertensive and type 2 diabetes mellitus patients. Nitric Oxide. 2007;16(3):348-55.

9. Zaros PR, Pires CE, Bacci M Jr, Moraes C, Zanesco A. Effect of 6-months of physical exercise on the nitrate/nitrite levels in hypertensive postmenopausal women. BMCWomens Health. 2009;19:9-17.

10. Claudino MA, Delbin MA, Franco-Penteado CF, Priviero FB, De Nucci G, Antunes E, et al. Exercise training ameliorates the impairment of endothelial and nitrergic corpus cavernosum responses in diabetic rats. Life Sci. 2011;31(88):272-7.

11. KimuraT, Yokoyama T, MatsumuraY,Yoshiike N, Date C, Muramatsu M, et al. NOS3 genotype-dependent correlation between blood pressure and physical activity. Hypertension. 2003;41(2):355-60.

12. Sarzynski MA, Rankinen $T$, Sternfeld $B$, Fornage $M$, Sidney $S$, Bouchard C. SNP-by-fitness and SNP-by-BMI interactions from seven candidate genes and incident hypertension after 20 years of follow-up: the CARDIA Fitness Study. J Hum Hypertens. 2011;25(8):509-18.

13. Rankinen T, Rice T, Pérusse L, Chagnon YC, Gagnon J, Leon AS, et al. NOS3 Glu298Asp genotype and blood pressure response to endurance training: the HERITAGE family study. Hypertension. 2000;36(5):885-9.

14. Negrao MV, Alves CR, Alves GB, Pereira AC, Dias RG, Laterza MC, et al. Exercise training improves muscle vasodilatation in individuals with T786C polymorphism of endothelial nitric oxide synthase gene. Physiol Genomics. 2010;42A(1):71-7.

15. Sponton $\mathrm{CH}$, Rezende $\mathrm{TM}$, Mallagrino PA, Franco-Penteado $\mathrm{CF}$, Bezerra MA, Zanesco A. Women with TT genotype for eNOS gene are more responsive in lowering blood pressure in response to exercise. Eur J Cardiovasc Prev Rehabil. 2010;17(6):676-81.

16. Esposti RD, Sponton $\mathrm{CH}$, Malagrino PA, Carvalho FC, Peres E, Puga GM, et al. Influence of eNOS gene polymorphism on cardiometabolic parameters in response to physical training in postmenopausal women. Braz J Med Biol Res. 2011;44(9):855-63.

17. Kline GM, Porcari JP, Hintermeister R, Freedson PS, Ward A, McCarron RF, et al. Estimation of VO2max from a one-mile track walk, gender, age, and body weight. Med Sci Sports Exerc. 1987;19(3):253-9.

18. Pulkkinen A, Viitanen L, Kareinen A, Lehto S, Vauhkonen I, Laakso $M$. Intron $4 \mathrm{~b} / \mathrm{a}$ polymorphism of the endothelial nitric oxide synthase gene is associated with elevated blood pressure in type 2 diabetic patients with coronary heart disease. J Mol Med. 2000;78(7):372-9.

19. IV Brazilian Guideline for Dyslipidemia and Atherosclerosis prevention. Arq Bras Cardiol. 2007;88(Suppl 1):2-19.

20. Cattaruzza M, GuzikTJ, Słodowski W, Pelvan A, Becker J, Halle M, et al. Shear stress insensitivity of endothelial nitric oxide syntha- se expression as a genetic risk factor for coronary heart disease. Circ Res. 2004;95(8):841-7.

21. Dosenko VE, Zagoriy VY, Haytovich NV, Gordok OA, Moibenko AA. Allelic polymorphism of endothelial NO-synthase gene and its functional manifestations. Acta Biochim Pol. 2006;53:299-302.

22. Wang $\mathrm{XL}$, Wang J. Endothelial nitric oxide synthase gene sequence variations and vascular disease. Mol Genet Metab. 2000;70:241-51.

23. Miyamoto $Y$, Saito $Y$, Nakayama $M$, Shimasaki $Y$, Yoshimura $T$, Yoshimura $\mathrm{M}$, et al. Replication protein $\mathrm{A} 1$ reduces transcription of the endothelial nitric oxide synthase gene containing a -786T$\rightarrow C$ mutation associated with coronary spastic angina. Hum Mol Genet. 2000;9(18):2629-37.

24. Tesauro M, Thompson WC, Rogliani P, Qi L, Chaudhary PP, Moss J. Intracellular processing of endothelial nitric oxide synthase isoforms associated with differences in severity of cardiopulmonary disease: cleavage of proteins with aspartate vs. glutamate at position 298. Proc Natl Acad Sci U S A. 2000;14(6):2832-5.

25. Lakoski SG, Barlow CE, Farrell SW, Berry JD, Morrow JR Jr, Haskell WL. Impact of body mass index, physical activity, and other clinical factors on cardiorespiratory fitness (from the Cooper Center longitudinal study). Am J Cardiol. 2011;108(1):34-9.

26. Gupta S, Rohatgi A, Ayers CR, Willis BL, Haskell WL, Khera A, et al. Cardiorespiratory fitness and classification of risk of cardiovascular disease mortality. Circulation. 2011;5(13):1377-83.

27. Aspenes ST, Nilsen TI, Skaug EA, Bertheussen GF, Ellingsen $\varnothing$, Vatten $\mathrm{L}$, et al. Peak oxygen uptake and cardiovascular risk factors in 4631 healthy women and men. Med Sci Sports Exerc. 2011;43(8):1465-73.

28. Keteyian SJ, Brawner CA, Savage PD, Ehrman JK, Schairer J, Divine $\mathrm{G}$, et al. Peak aerobic capacity predicts prognosis in patients with coronary heart disease. Am Heart J. 2008;156(2):292-300.

29. Han Y, Xu W, Zhang W, Liu N, JiY.T-786C polymorphism in the endothelial nitric oxide synthase gene is associated with increased risk of coronary artery disease in a Chinese population. Pharmacology. 2010;85(4):211-6.

30. Hoffmann IS, Tavares-Mordwinkin R, Castejon AM, Alfieri AB, Cubeddu LX. Endothelial nitric oxide synthase polymorphism, nitric oxide production, salt sensitivity and cardiovascular risk factors in Hispanics. J Hum Hypertens. 2005;19(3):233-40.

31. Imamura A, Takahashi R, Murakami R, Kataoka H, Cheng XW, Numaguchi $Y$, et al. The effects of endothelial nitric oxide synthase gene polymorphisms on endothelial function and metabolic risk factors in healthy subjects: the significance of plasma adiponectin levels. Eur J Endocrinol. 2008;158(2):189-95.

32. Joshi MS, Mineo C, Shaul PW, Bauer JA. Biochemical consequences of the NOS3 Glu298Asp variation in human endothelium: altered caveolar localization and impaired response to shear. FASEB J. 2007;21(11):2655-63.

33. Zanesco A, Silva CP, Delbin MA, La Guardia PG, Davel AP, Priviero FB. Mitochondrial biogenesis and redox state in gastrocnemius muscle from trained rats supplemented with L-arginine. Free Radic Biol Med. 2011;51(Suppl 1).

34. Frayn KN, Fielding BA, Karpe F. Adipose tissue fatty acid metabolism and cardiovascular disease. Curr Opin Lipidol. 2005;16(4):409-15. 